\title{
Expanded use of antenatal steroid and the neonate in NICU
}

Volume 4 Issue 6 - 2016

Abbreviations: ANS, antenatal steroids; RDS, respiratory distress syndrome; IVH, intraventricular hemorrhage; NEC, necrotizing enterocolits; PDA, patent ductus arteriosus; BPD, bronchopulmonary dysplasia; ALPS, antenatal late preterm steroids

\section{Introduction}

The outcomes of prematurely born infants subjected to the neonatal intensive care have improved significantly with the introduction of antenatal steroids (ANS) and postnatal exogenous surfactant therapies. The addition of ANS has reduced neonatal morbidity and mortality significantly more than the use of exogenous surfactant alone.

In utero exposure to ANS results in structural and functional enhancement of multiple organ systems in the neonate and is shown to be safe for the mother. Besides the well documented beneficial effects on mortality, respiratory distress syndrome (RDS), intraventricular hemorrhage (IVH) and necrotizing enterocolits (NEC) - all of which are reduced by about $50 \%$, significant decrease in systemic sepsis in the first $48 \mathrm{hrs}$ of life, as well as in the level of NICU care and respiratory support requirements have been reported with the use of ANS. ${ }^{1}$ In addition, ANS exposure causes extenuation of hyperkalemia and hypotension, both of which are associated with mortality and other outstanding complications in the extremely premature neonates. The genomic effects of steroids reverse the lysosomal destruction of down regulated adrenal receptors brought about by the critical illness like RDS, and inhibit the rate limiting enzyme catechol-0methyltransferase in catecholamine metabolism, which result in increased local and systemic catecholamine concentrations and stabilization of blood pressure in the sick hypotensive extremely low birth weight infants. ${ }^{2}$ Biochemically, ANS accelerates the pulmonary anti oxidant enzymes production, induces $\mathrm{Na}$ - permeable- ion channels in lungs and enhances $\mathrm{Na}+\mathrm{K}+\mathrm{ATPase}$ in multiple organs, including lungs, brain, colon and kidneys. ${ }^{1,3}$ These effects have significant implications in the fetal and immediate postnatal lung water clearance and general electrolytes and water metabolism, which are protective for morbidities like RDS, patent ductus arteriosus (PDA), IVH and prolonged oxygen dependence. ANS has profound effects on surfactant metabolism, starting from enhancement of gene expression to exocytosis of surfactant particles into the alveolar space. Cytostructurally, ANS decreases the thickness of pulmonary interstitium, improves pulmonary vascular integrity and increases the gas exchange surface area in the premature lungs. It enhances the functional residual volume, improves lung mechanics by virtue of significantly increasing passive respiratory compliance and decreases the work of breathing, thereby supporting oxygenation and ventilation. Its effects on vascular integrity protect against the pathogenesis of IVH and NEC.

ANS is given as betamethasone via an intramuscular dose of 12 $\mathrm{mg}$, repeated $24 \mathrm{hrs}$ later, to mothers who are in preterm labor during 24 and 34 weeks of gestation with delivery anticipated within 7 days

\section{Rita PVerma}

Department of Pediatrics, Nassau University Medical Center, USA

Correspondence: Rita P.Verma, MD, FAAP, Attending Neonatologist, Director of Research, Department of Pediatrics, Nassau University Medical Center, State University of New York, 220 I Hempstead Turnpike, East Meadow, NY I I554, USA, Tel 631-875-5375, Email ritaverma@aol.com

Received: May 09, 2016 | Published: June 20, 2016

of treatment. ${ }^{4}$ Alternatingly, dexamethasone in four doses of $6 \mathrm{mg}$ each may be given intramuscularly 12 hours apart. If betamethasone and dexamethasone are not available, hydrocortisone $500 \mathrm{mg}$ given intravenously every 12 hours for 4 doses can be used. There is no definite evidence supporting superiority of betamethasone over dexamethasone. ${ }^{5}$ Adverse neurological outcomes have been less frequently associated with betamethasone. Dexamethasone has a higher affinity for steroid receptors and it is demonstrated to decrease the incidence of IVH more than betamethasone. Betamethasone causes phospholipase 2 expression inhibition through a reduction in tumor necrosis factor in the pulmonary tissue of newborn rats more vigorously than dexamethasone, which may characterizes this glucocorticoid as a better choice than dexamethasone. ${ }^{6}$

The recommended time frame of ANS administration is $24 \mathrm{hrs}$ to 7 days before delivery for maximum fetal advantage. ${ }^{4}$ This is met in only $12-25 \%$ of mothers due to the unpredictability of circumstances. It can be reportedly increased up to $50 \%$ if the doses are given at 12 hours interval. No advantages in terms of reduction in neonatal death, RDS or IVH are noted beyond 7 days of administration of ANS, whereas, an incomplete course with a single dose has been shown to reduce the need for vasopressors, as well as the occurrences of intraventricular hemorrhage and neonatal death. The minimal interval from fetal exposure to corticosteroids to delivery for improved postnatal lung function has been reported to be between 8 and 15 hours. The corticosteroid effects on pulmonary edema and blood pressure can be demonstrated within 8 hours of administration. ${ }^{6}$

According to two meta analyses, the gestational age group which benefits the most from the intervention in regards to mortality, RDS, IVH and NEC is that of between 26 and 34 weeks. ${ }^{7,8}$ Although the treatment guidelines recommend ANS administration in preterm labor between 24-34 weeks of gestation, some positive effects are demonstrated below 24 weeks, making ANS a potentially valid intervention at this stage of maturation. A meta analysis of randomized trials involving ANS therapy before 24 weeks of gestation revealed that at 23 weeks there is a reduction in death, but not in RDS, IVH or NEC, whereas, at 22 weeks only a non significant reduction in perinatal mortality is noted (OR $0.6,95 \%$ CI $0.4-1.07) .{ }^{9}$ In another study both mortality and NEC were reduced in ANS exposed neonates 
at 22 weeks but neurodevelopmental outcome at 18-22 weeks of adjusted postnatal life was not different from those who were unexposed. ${ }^{10}$ When analyzing infants born between 22-25 weeks as a group, the same study demonstrated a decrease in short term death, IVH and PVL but not in bronchopulmonary dysplasia (BPD) or NEC. Intact survival was shown to double but stayed low at $36 \%$ with ANS in this cohort. Furthermore, both death and neurodevelopmental outcomes were improved at 18-22 weeks of adjusted postnatal age in this gestational age group. Exposure to a full course of ANS reduced mortality by $82 \%$ in a retrospective cohort of infants born at 23 weeks of gestation but showed no effects on the occurrences of necrotizing enterocolitis or severe intraventricular hemorrhage. ${ }^{11}$ It is calculated that 7 to 9 infants born at 23 to 25 weeks of gestational age need to be treated with ANS in order to prevent 1 death. A report involving ANS therapy in a prospective cohort of over 4 thousand infants between $22^{0 / 7}$ and $25^{0 / 7}$ weeks of gestation showed that the risk of death and any degree of neurodevelopmental impairment are reduced at 18-22 months of corrected age among survivors. ${ }^{12}$ These advantages were equivalent to a 1-week increase in gestational age. When evaluating long term outcomes, the prospective cohort EPICure study, which involved mothers with $<26$ weeks of gestation reported ANS to be associated with an increased mental development index at 2.5 and at 6 years of age. ${ }^{13}$ These results suggest that the positive effects of ANS administration are clearly demonstrated by 23 weeks of gestation onwards but are less certain at 22 weeks or less. The caveat is that gestational age cannot be established with certainty at 22 weeks and a discrepancy of as much as 2 weeks may be encountered at the stage of maturation, which makes it a critically gray zone for equitable and precise decision making. ANS is recommended to be given before 22 weeks only if the delivery is anticipated within a week, and under strong parental wish for aggressive resuscitation and postnatal management despite an in depth evidence based consultation with the maternal-fetal medicine and neonatology specialists. ${ }^{14}$ During these discussions the parents should be extensively counseled about the possibility of a severely compromised survival, as well as a strong likelihood of short or long term mortality despite the intervention. The ethics and legal personnel should be involved in the discussions as appropriate by the institutional standards in order to avoid any future incrimination of wrongful life when giving ANS at the lowest controversial limit of viability.

There have been active discussions about the use of ANS beyond the traditional upper limit of 34 weeks and research has been conducted to assess the role of ANS therapy in mothers who are in late preterm or early term gestations. The Antenatal Late Preterm Steroids Trial (ALPS) demonstrated that ANS reduces the need for CPAP, high flow nasal canula and surfactant, as well as the occurrences of transient tachypnea of newborn and BPD in infants at 34-36 weeks of gestation, but has no effects on RDS or the need for mechanical ventilation in them..$^{15}$ In a recent study Gyamphi Bammernan et al. ${ }^{15}$ reported a reduction in acute respiratory diseases, surfactant use and BPD, with an increase in the occurrence of neonatal hypoglycemia in infants whose mothers were treated with betamethasone at 34-36 weeks of gestation. ${ }^{16}$ In regards to the infants at gestational age of $\geq 37$ weeks the Antenatal Steroids for Term Caesarean Section trial (ASTECS), which administered steroids 48 hours before scheduled cesarean delivery during the $38^{\text {th }}$ week of gestation, reported a reduction in admission to NICU for the composite occurrence of transient tachypnea of the newborn and respiratory distress. ${ }^{17}$ The study further reported that steroid-exposed children were in the lowest achievement group compared with the control group at 8 to 15 years of age. Recently,
Ahmed et al. ${ }^{18}$ demonstrated a decrease in respiratory morbidities in infants with maternal betamethasone intake prior to elective cesarean section at 38 weeks of gestation. ${ }^{18}$ Based on the evidence coming from these trials, the Society for Maternal-Fetal Medicine Specialists has recently approved a two-dose course of antenatal betamethasone for women at $34^{0 / 7}$ to $36^{6 / 7}$ weeks of gestation who are at high risk for preterm delivery within seven days, provided certain conditions are met. These conditions are specified as: preterm labor with cervical dilation $\geq 3 \mathrm{~cm}$ or effacement $\geq 75$ percent before treatment, no tocolysis to delay delivery for administering full course of ANS, which should not be administered until a definite plan for delivery is made in the presence of medical or obstetric indications for delivery ${ }^{19}$ The American College of Obstetricians and Gynecologists endorses ANS in women with a singleton pregnancy at $34^{0 / 7}$ to $36^{6 / 7}$ weeks of gestation who are at risk of preterm birth within 7 days in the absence of chorioamnionitis, with no tocolysis to delay the delivery for ANS and no interference with medically or obstetrically indicated preterm delivery decisions. Additionally, they contraindicated a repeat course of ANS if the mother had already received one and recommended close monitoring of newborn for hypoglycemia born with ANS exposure. ${ }^{4}$ The Royal College of Obstetricians and Gynaecologists recommends routine administration of ANS in women who are at risk for preterm birth up until the end of $34^{6 / 7}$ weeks, and in those mothers who must undergo scheduled cesarean delivery before $39^{107}$ weeks of gestation. ${ }^{20}$

The routine use of ANS in late preterm pregnancy is not universally recommended due to concerns about the adverse effects of ANS on the developing neurological and endocrinal system of the fetus, which could potentially have significant short and long term neurological and metabolic consequences in infancy or in later life. ${ }^{21}$ Steroids are shown to alter neuronal migration, synaptic plasticity and neurotransmitter activities in the developing fetal brain. In late preterm or early term fetus axonal proliferation and outgrowth, as well as the dendritic synapses are in the process of development with rapid mitotic cellular activities. ANS exposure at this stage might accelerate apoptosis and cell death with resultant restriction of neurons growth and function, as evidenced in animal studies. Neural and glial proliferation, neuronal migration and myelination take place during the earlier gestational months and antenatal steroid exposure might delay these critical processes. Multiple courses of steroids are shown to accentuate these adverse effects in animal models and therefore, are not recommended except for in some specific well defined critical conditions.

The full extent of the expanded use of ANS in neonates is still not completely realized. Whereas, the administration of ANS during the periviable period of $23^{0 / 7}$ to $25^{0 / 7}$ weeks is now considered the standard of care, more information is awaited regarding the long term endocrinal, neurological and metabolic sequelae before the intervention can be considered universally applicable beyond 34 weeks of pregnancy. As the incidence is low and the acuity and nature of respiratory illnesses relatively milder, widespread use of antenatal steroids at this stage might result in unnecessary medication and avoidable exposure to potentially serious long lasting complications in the treated infants. Even though the impact of ANS administration is not yet fully defined, the fact remains that ANS has significantly improved the outcomes of the tiniest neonates in the NICU and has emerged as one of the major cornerstones of the contemporary neonatal care.

\section{Acknowledgments}

None. 


\section{Conflicts of Interset}

None.

\section{References}

1. Wapner R, Jobe AH. Controversy: Antenatal Steroids. Clin Perinatol. 2011;38(3):529-545.

2. Seri I, Tan R, Evans J. Cardiovascular Effects of Hydrocortisone in Preterm Infants with Pressor-Resistant Hypotension. Pediatrics. 2001;107(5):1070-1074.

3. Verma R, Horvath $\mathrm{K}$, Lu RB. Perinatal colonic and renal $\mathrm{Na}+\mathrm{K}+\mathrm{ATPase}$ activity in response to potassium load and betamethasone. $J$ Clin and Lab Med. 1994;123(5):676-683.

4. ACOG Committee on Obstetric Practice. ACOG Committee Opinion No. 475: antenatal corticosteroid therapy for fetal maturation. Obstet Gynecol. 2001;117(2 Pt 1):422-424.

5. Brownfoot FC, Gagliardi DI, Bain E, et al. Different corticosteroids and regimens for accelerating fetal lung maturation for women at risk of preterm birth. Cochrane Database Syst Rev. 2001;8:CD006764.

6. Romejko-Wolniewicz E, Teliga-Czajkowska J, Czajkowski K. Antenatal steroids: can we optimize the dose? Curr Opin Obstet Gynecol. 2004;26(2):77-82.

7. Roberts D, Dalziel S. Antenatal corticosteroids for accelerating fetal lung maturation for women at risk of preterm birth. Cochrane Database Syst Rev. 2003;3:CD004454.

8. Onland W, de Laat MW, Mol BW, et al. Effects of antenatal steroid given prior to 26 weeks of gestation: a systematic review of randomizrd controlled trial. Am J Perinatol. 2011;28(1):33-44

9. Park CK, Isayama T, McDonald SD. Antenatal Corticosteroid Therapy before 24 Weeks of Gestation: A Systematic Review and Meta-analysis. Obstet Gynecol. 2016;127(4):715-725.

10. Carlo WA, McDonald SA, Fanaroff AA, et al. Association of antenatal corticosteroids with mortality and neurodevelopmental outcomes among infants born at 22 to 25 weeks' gestation. JAMA. 2011;306(21):2348-2358
11. Hayes EJ, Paul DA, Stahl GE, et al. Effect of antenatal corticosteroids on survival for neonates born at 23 weeks of gestation. Obstet Gynecol. 2008;111(4):921-926.

12. Tyson JE, Parikh NA, Langer J, et al. Intensive care for extreme prematurity-moving beyond gestational age. $N$ Engl $J$ Med. 2008;358(16):1672-1681.

13. Costeloe K, EPICure Study Group. EPICure: facts and figures: why preterm labour should be treated. BJOG. 2006;113(Suppl 3):10-12.

14. Raju TNK, Mercer BM, Burchfield DJ, et al. Periviable birth: executive summary of a Joint Workshop by the Eunice Kennedy Shriver National Institute of Child Health and Human Development, Society for MaternalFetal Medicine, American Academy of Pediatrics, and American College of Obstetricians and Gynecologists. J Perinatol. 2001;34(5):333-342.

15. Gyamfi-Bannerman C. 1: Antenatal Late Preterm Steroids (ALPS): a randomized trial to reduce neonatal respiratory morbidity. AJOG. 2016;214(Suppl 1):S2.

16. Gyamfi-Bannerman C, Thom EA, Blackwell SC, et al. Antenatal Betamethasone for Women at Risk for Late Preterm Delivery. $N$ Engl J Med. 2016;374(14):1311-1320.

17. Stutchfield P, Whitaker R, Russell I. Elective Caesarean Section (ASTECS) Research Team. Antenatal betamethasone and incidence of neonatal respiratory distress after elective caesarean section: pragmatic randomised trial. $B M J .2005 ; 331(7518): 662$

18. Ahmed MR, Sayed Ahmed WA, Mohammed TY. Antenatal steroids at 37 weeks, does it reduce neonatal respiratory morbidity? A randomized trial. J Matern Fetal Neonatal Med. 2015;28(12):1486-1490.

19. Society for Maternal-Fetal Medicine (SMFM) Publications Committee. Implementation of the Use of Antenatal Corticosteroids in the Late Preterm Birth Period in Women at Risk for Preterm Delivery. Am J Obstet Gynecol. 2016;pii: S0002-9378(16):00475-00480.

20. Antenatal corticosteroids to reduce neonatal morbidity and mortality (Green-top Guideline no. 7). Royal College of Obstetricians and Gynaecologists. 2010.

21. Elizabeth Asztalos. Antenatal Corticosteroids: A Risk Factor for the Development of Chronic Disease. Journal of Nutrition and Metabolism. 2012;(2012):9. 